\section{Retinal changes associated with type 2 glomerulonephritis}

CE McAvoy and G Silvestri

Eye (2005) 19, 985-989. doi:10.1038/sj.eye.6701697; published online 17 September 2004

Aims The aims of this study were to:

1. Investigate retinal changes associated with type 2 membranoproliferative glomerulonephritis (type 2 MPGN).

2. Assess whether there was a relationship between the severity of the kidney disease and ophthalmoscopically visible fundal changes.

3. Find out if renal transplantation was linked with visual deterioration.

4. Decide if patients with type 2 MPGN need to be referred for ophthalmological assessment.

Methods The patients were identified from pathology department records as having renal biopsy proven type 2 MPGN from January 1981 to October 2003. Patients were invited to attend for ophthalmic assessment.

Results A total of 26 biopsy proven cases of type 2 MPGN were identified. One patient lost vision from a possible occult choroidal neovascular membrane. The extent of ocular involvement did not consistently appear to be related to the severity of the renal involvement, although there was a relationship between the presence of ocular lesions and the duration of the disease. The longer the disease was present the more likely the patients were to have ophthalmoscopically visible fundal changes. Renal transplantation did not appear to have a detrimental effect on vision with the possible exception of one patient.

Conclusion Referral of type 2 MPGN patients by the renal physician for an initial ophthalmological assessment may be beneficial so that symptoms of choroidal neovascularization can be explained and patients advised to seek urgent help if distortion of central vision occurs. Renal transplantation does not appear to be a risk factor for the development of choroidal neovascular membranes.
Keywords: type 2 membranoproliferative glomerulonephritis; dense deposit disease; drusen; renal transplantation; choroidal neovascularization; posterior chorioretinopathy

\section{Introduction}

Type 2 membranoproliferative glomerulonephritis (MPGN), also known as dense deposit disease, is a chronic glomerulonephritis that occurs in older children and adults. ${ }^{1}$ Three distinct types are identifiable based on light and electron microscopic differences. ${ }^{1}$ Duval-Young et $a l^{2,3}$ described for pigment epithelial (RPE) alterations and dense deposit disease. They demonstrated that the electron microscopic appearances of the deposits in the kidney and the eye are identical. ${ }^{2,3}$ In the basement membrane of the RPE, these electron dense deposits ophthalmoscopically resemble drusen and can be associated with RPE alterations. ${ }^{4-6}$ In advanced stages, RPE detachments and choroidal neovascularization can occur, which may lead to loss of central visual acuity. ${ }^{5}$ Similar fundal changes have not been observed in type 1 or type 3 MPGN. ${ }^{3-6}$ Therefore, the association between kidney disease and typical ocular lesions may help renal physicians make the diagnosis in those with unclassified MPGN.

\section{Materials and methods}

All the patients were identified from pathology department records as having renal biopsy proven type 2 MPGN between January 1981 and October 2003. All patients underwent full ophthalmological examination including Snellen visual acuity measurement, slit-lamp the first time in 1989 the association of retinal
Department of Ophthalmology Royal Victoria Hospital Belfast

Northern Ireland UK

Correspondence: CE McAvoy Department of Ophthalmology Royal Victoria Hospital Grosvenor Road Belfast BT12 6BA Northern Ireland UK

Tel: +442890240503 ext 3822

Fax: + 442890330744

E-mail: claramcavoy@ doctors.org.uk.

Received: 30 March 2004 Accepted: 13 July 2004 Published online: 17 September 2004 
biomicroscopy, and fundoscopy. In addition, a short questionnaire relating to their medical and ophthalmic history, kidney disease, and medication was completed.

\section{Results}

A total of 26 biopsy proven cases of type 2 MPGN were identified. There were 19 female and seven male patients, two female and one male patient(s) were deceased. Two patients were not contactable. The 21 remaining patients were invited to attend for clinical examination. In all, 20 patients attended. Table 1 summarizes the salient details.

The onset of renal disease ranged from 4 to 33 years (mean 14.4 years). The age at time of ocular examination varied from 9 to 57 years (mean 29.1 years). The length of time of renal disease ranged from 4 to 35 years (mean 15.3 years). The course of the renal disease varied. Eight patients had undergone kidney transplantation and in five patients the first transplant was still functioning. One patient had had two transplants and two patients had had one transplant that had failed, and now both were receiving haemodialysis. Two other patients were on peritoneal dialysis. The other 11 patients were under observation.
Only three patients had been seen in the ophthalmology department prior to attending for screening two of these with complaints unrelated to their kidney disease. These two patients had been seen 6 and 7 years earlier both had maintained good visual acuity with little change noted in fundal appearances.

The third patient deserves a more detailed analysis. This was patient 2 whose question 'Could transplantation affect my sight?' prompted the study. He had first presented to the renal physicians in 1989 with severe hypertension, proteinuria, and a creatinine level of 223. A renal biopsy at that time showed findings typical of type 2 MPGN. He was commenced on haemodialysis and eventually switched to chronic ambulatory peritoneal dialysis. In 1992, he underwent his first renal transplant, but 2 years later presented with haematuria, proteinuria, and a creatinine level of 297. Repeat biopsy showed recurrent disease with virtually no rejection. In 1996, he had a second renal transplant and in 1998, this was destroyed by recurrent disease.

In 1998, he presented to eye casualty with a reduction of vision in his left eye. This had become much worse over the preceding few days, but he felt vision had been deteriorating over several months. On examination,

Table 1 Clinical summary of the 20 patients examined

\begin{tabular}{|c|c|c|c|c|c|c|}
\hline \multirow{2}{*}{$\begin{array}{l}\text { Patient, age } \\
\text { (years), sex }\end{array}$} & \multirow{2}{*}{$\begin{array}{l}\text { Age onset } \\
\text { (years) }\end{array}$} & \multirow{2}{*}{$\begin{array}{l}\text { Disease duration } \\
\text { (years) }\end{array}$} & \multirow{2}{*}{$\begin{array}{l}\text { Course of renal } \\
\text { disease }\end{array}$} & \multicolumn{2}{|c|}{ Vision } & \multirow[t]{2}{*}{ Macular and fundal changes } \\
\hline & & & & Right & Left & \\
\hline 1. $28, \mathrm{~F}$ & 11 & 17 & Transplant & $6 / 6$ & $6 / 6$ & BLD macula and periphery \\
\hline 2. $53, \mathrm{M}$ & 39 & 24 & $\begin{array}{l}\text { Transplant } \times 2 \\
\text { haemodialysis }\end{array}$ & $6 / 12$ & $\mathrm{HM}$ & $\begin{array}{l}\text { Atr. macula, BLD (right), } \\
\text { Exudative detachment (left) }\end{array}$ \\
\hline 3. $33, \mathrm{~F}$ & 15 & 18 & Transplant & $6 / 18^{\mathrm{a}}$ & $6 / 6$ & BLD macula only \\
\hline 4. $57, \mathrm{~F}$ & 33 & 24 & Observation & $6 / 6$ & $6 / 9^{a}$ & $\begin{array}{l}\text { Atr. macula with BLD macula and } \\
\text { periphery }\end{array}$ \\
\hline 5. $39, \mathrm{~F}$ & 19 & 20 & Observation & $6 / 6$ & $6 / 6$ & SD macula and periphery \\
\hline 6. $23, \mathrm{M}$ & 13 & 10 & Observation & $6 / 6$ & $6 / 6$ & RPE changes macula only \\
\hline 7. $27, \mathrm{~F}$ & 14 & 13 & $\begin{array}{l}\text { Transplant } \times 1 \\
\text { haemodialysis }\end{array}$ & $6 / 6$ & $6 / 6$ & BLD macula and periphery \\
\hline 8. $34, \mathrm{~F}$ & 12 & 22 & Transplant & $6 / 6$ & $6 / 6$ & SD macula, BLD periphery \\
\hline 9. $37, \mathrm{~F}$ & 15 & 22 & Observation & $6 / 5$ & $6 / 5$ & BLD macula and periphery \\
\hline 10. $22, \mathrm{~F}$ & 8 & 14 & Observation & $6 / 9$ & $6 / 6$ & RPE changes and Atr. Macula \\
\hline 11. $47, \mathrm{~F}$ & 12 & 35 & Transplant & $6 / 9$ & $6 / 9$ & No macular changes, BLD \\
\hline 12. $33, \mathrm{~F}$ & 23 & 10 & Observation & $6 / 9$ & $6 / 9$ & RPE change macula only \\
\hline 13. $28, \mathrm{M}$ & 13 & 15 & Transplant & $6 / 5$ & $6 / 5$ & SD macula, BLD \\
\hline 14. $27, \mathrm{~F}$ & 8 & 19 & Peritoneal dialysis & $6 / 9$ & $\mathrm{CF}^{\mathrm{b}}$ & Atr. macula, BLD \\
\hline 15. $30, \mathrm{M}$ & 16 & 14 & Observation & $6 / 5$ & $6 / 5$ & BLD macula and periphery \\
\hline 16. $45, \mathrm{M}$ & 16 & 29 & $\begin{array}{l}\text { Transplant } \times 1 \\
\text { haemodialysis }\end{array}$ & $6 / 6$ & $6 / 6$ & No macular changes, BLD \\
\hline 17. $9, \mathrm{~F}$ & 4 & 5 & Observation & $6 / 6$ & $6 / 6$ & No fundal changes \\
\hline 18. $12, \mathrm{~F}$ & 9 & 3 & Observation & $6 / 6$ & $6 / 6$ & No fundal changes \\
\hline 19. $12, \mathrm{~F}$ & 6 & 6 & Peritoneal dialysis & $6 / 6$ & $6 / 6$ & No macular changes, BLD \\
\hline 20. $17, \mathrm{~F}$ & 16 & 1 & Observation & $6 / 18^{a}$ & $6 / 6$ & No fundal changes \\
\hline
\end{tabular}

$\mathrm{BLD}=$ basal laminar drusen; Atr. = atrophy; $\mathrm{SD}=$ soft drusen; RPE = retinal pigment epithelial.

${ }^{a}$ Amblyopic eye not related to renal problem.

bPatient was noted to have bilateral papilloedema and macular stars in keeping with malignant hypertension at initial hospital visit. The left eye was left with poor visual acuity following this from optic nerve and macular damage. 

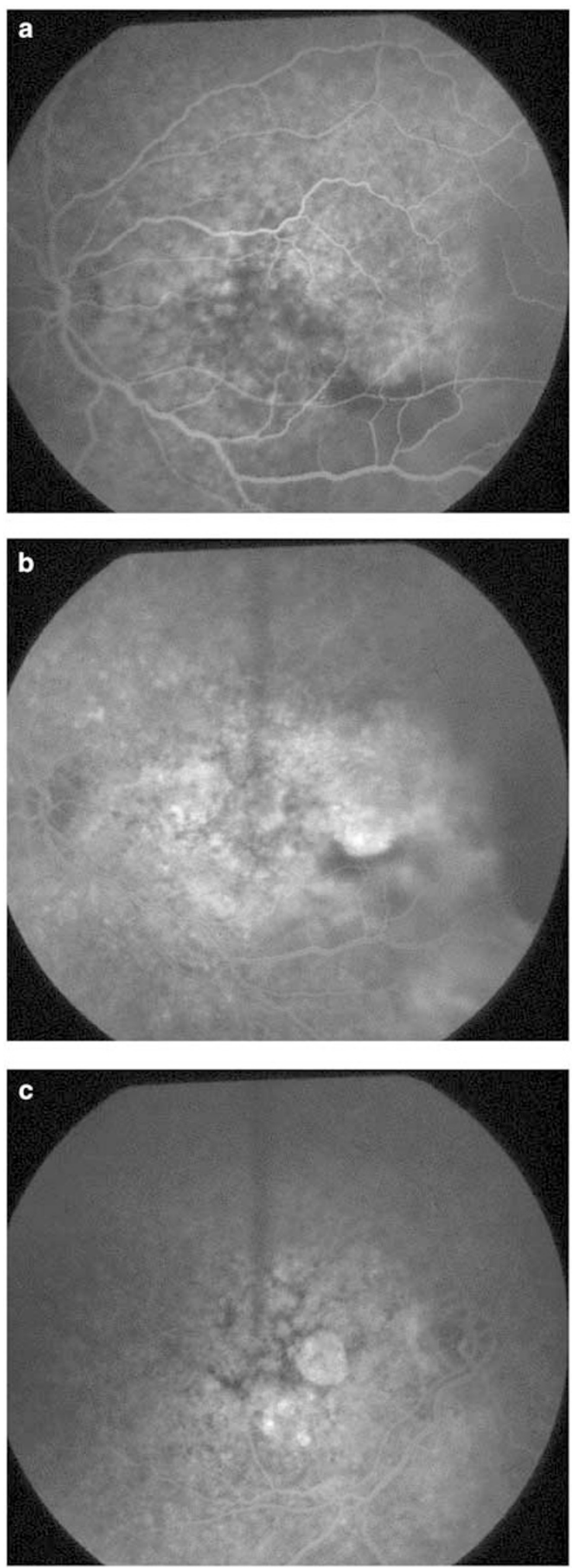

Figure 1 Fluorescein angiogram showing (a) exudative retinal detachment and (b) fluorescein leakage inferotemporal to the macula left eye in patient 2 in 1998 indicating possible occult choroidal neovascularization. (c) Fluorescein angiogram illustrating dry macular degeneration in patient 2 in 1998.
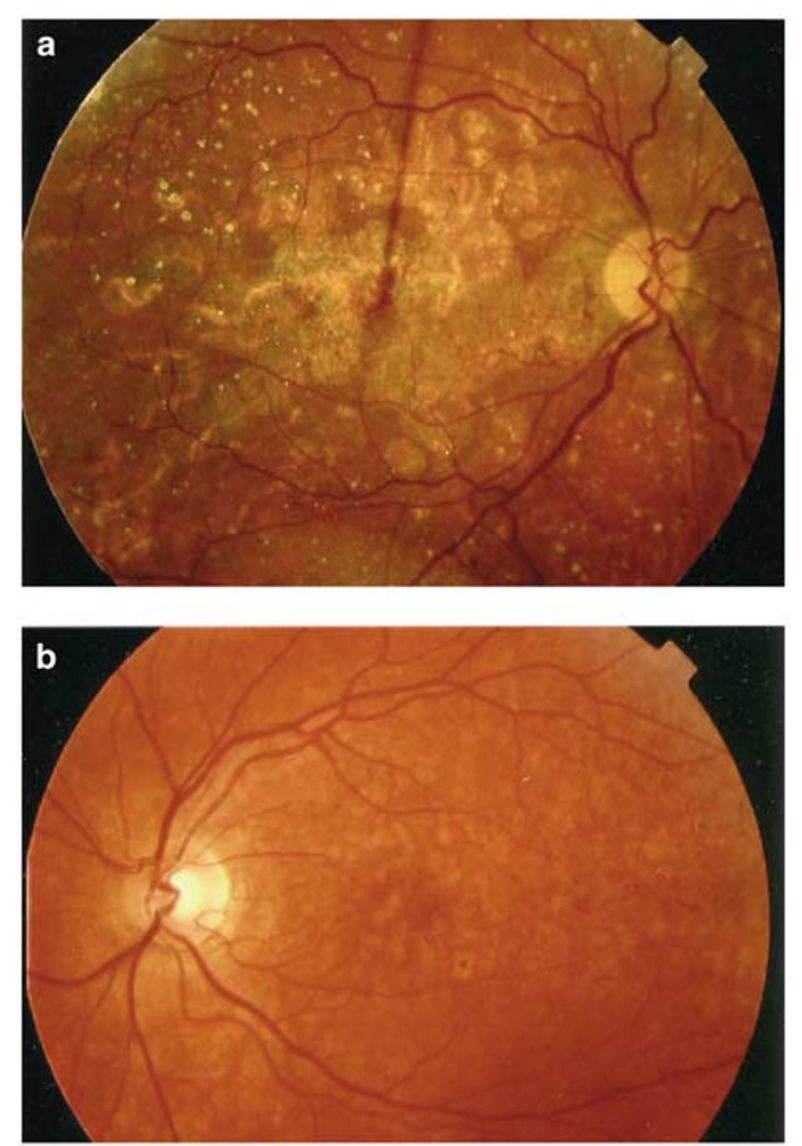

Figure 2 (a) Basal laminar drusen and atrophic maculopathy in patient 2 in 2003. (b) Soft drusen in patient 5 .

visual acuity was 6/9 right and hand movements left. Anterior segment examination was normal but dilated fundal examination revealed widespread drusen-like deposits in both eyes, with possible subretinal neovascularization and a marked exudative detachment in the left eye. There was no recent history of accelerated hypertension. Fluorescein angiography (Figure 1a-c) confirmed the clinical findings that were felt to be consistent with an occult choroidal neovascular membrane (CNVM), although another possibility could have been posterior chorioretinopathy associated with organ transplantation. ${ }^{7}$

In this study, ophthalmoscopy on all adults revealed the presence of either macular or fundal changes in keeping with type 2 MPGN. All adult patients had macular changes except perhaps surprisingly patients 11 and 16, who despite having renal disease for 35 and 29 years, respectively, and undergoing renal transplants, did not show any evidence of macular problems, although both were noted to have multiple drusen scattered throughout the fundus. In the adults in this series, the commonest macular findings were drusen extending temporally from the maculae sometimes in association 
with RPE hypertrophy and atrophy. Only one patient developed signs of a possible occult CNVM. Ophthalmoscopically visible mid-peripheral and peripheral fundal changes were not universal being absent in four of the patients. Fundal changes varied from the small discrete so-called 'basal laminar drusen' (Figure 2a) to more numerous larger 'soft drusen' (Figure $2 b$ ) in the mid-peripheral and peripheral retina that were symmetrical between both fundi. The peripheral 'basal laminar drusen' often had a whitish center surrounded by hyperpigmentation, whereas others appeared as innumerable small yellow dots.

Of the four children in the study, none had macular changes and only one had evidence of the peripheral drusen just described. This was patient 17 who had the most severe renal involvement and had been diagnosed with type 2 MPGN for 6 years. In a study by Leys $e t a l^{4}$ in Paediatric Nephrology, $3 / 5$ patients with type 2 MPGN had ocular involvement, although it was commented that the small yellow nodules associated with the condition were difficult to identify by ophthalmoscopy unless grouped in clusters. Fluorescein angiography enables a more detailed analysis and is essential for the detection of early changes.

In this series, the overall trend was that the extent of ocular involvement was not always comparable to severity of renal involvement and that transplantation, with the possible exception of patient 2, did not appear to have a detrimental effect on visual functioning. Unfortunately, it is not possible to say with absolute certainty that the three patients who died secondary to their kidney disease did not have visual problems. However, no record of them ever attending an ophthalmologist was found in their notes or reference made to any eye complaint. It did appear from this study that there was a relationship between the intensity of the ocular lesions and the apparent duration of the renal disease in that adults had more advanced ocular changes.

\section{Discussion}

Type 2 MPGN is an uncommon renal condition associated with electron dense deposits in the lamina densa of the glomerular basement membrane with C3 found in the capillary loops and mesangium. ${ }^{1}$ It is an aggressive disease often giving rise to end-stage renal failure in 6-10 years. ${ }^{1,5}$ Type $2 \mathrm{MPGN}$ is said to have a nearly equal sex distribution; however, this study found a preponderance of female patients (almost $3: 1$ ). ${ }^{1}$ The systemic nature of type 2 MPGN is illustrated by the fact that the electron dense deposits may also be found in the spleen, choriocapillaris, and Bruchs membrane of the eye and by the fact that the disease has a tendency to recur in allografts. ${ }^{5,6}$ The exact nature of the dense deposits found in type 2 MPGN are unknown, although recent data suggest that these deposits are compositionally similar but not identical to drusen. ${ }^{8,9}$ At present, no one is quite sure why there is such a range of clinical expression of the disease in both ocular and renal aspects. ${ }^{5}$ The deposits in the basement membrane can lead to a breakdown of the blood-retinal barrier by interfering with the RPE layer, and type 2 MPGN has been described in association with central serous retinopathy. ${ }^{10,11}$

Fundal changes range from scattered small 'basal laminar drusen' to more extensive 'soft' drusen involving the posterior pole that spread up to and beyond the equator often associated with pigmentary changes. As time goes on the development of atrophic changes in the retina and RPE is increasingly common. Macular changes range from small to large drusen-like deposits associated with RPE alterations with patients retaining excellent visual acuity to the development of retinal pigment epithelial detachments, choroidal neovascular membranes and visual loss. ${ }^{9,10,12}$

It is clear from our study, one of the largest to date, that type 2 MPGN-related retinopathy is a slowly progressive condition rarely giving rise to visual symptoms. The longer the condition is present, the more likely fundal changes are to be present. In this study, only one patient experienced a visual problem related to type 2 MPGN retinopathy, although as alluded to earlier, his renal transplantation may have had a part to play. Gass $\mathrm{et}^{\mathrm{al}} \mathrm{l}^{7}$ first described in 1992 four patients with segmental areas of RPE derangement in the posterior fundus associated with a secondary exudative detachment in 3 . The onset of visual symptoms in their patients were 5, 18, 36 months after renal transplantation and 12 months after heartlung transplantation. The authors hypothesized that local intravascular coagulation affecting the posterior choroid induced by subclinical graft rejection may have been the cause of the patients' ocular symptoms. It is uncertain what role, if any, transplantation has to play in visual deterioration in type 2 MPGN given that the seven other patients in this study did not experience any problems.

In conclusion, it is felt that unlike recommendations made by other studies (given the rarity of ocular complications associated with the disease) regular screening of type 2 MPGN patients should not be necessary. ${ }^{5,6,12,13}$ Currently, there is no literature directly commenting on the risk kidney transplantation poses to vision in this group of patients. This study suggests that the risk is minimal with only one patient out of eight experiencing visual problems possibly directly attributable to transplantation. In addition, this study emphasizes that there are differences in terms of severity of the disease between the eye and the kidney. Researchers have drawn parallels between the choriocapillaris/Bruchs membrane/RPE complex and the renal glomerulus in its response to type 2 MPGN; 
however, from this paper it can be seen that some patients may experience severe renal problems requiring kidney transplantation and yet show no or minimal retinal changes. Conversely, some patients who are only being observed from the renal point of view show significant eye involvement. The explanation for these findings is unknown but serves to illustrate that the extent of ocular involvement is not always in line with severity of renal disease.

\section{Acknowledgements}

I would like to thank Dr CM Hill, retired consultant pathologist who histologically diagnosed most of the cases described and Dr JL Best, who provided clinical information on patient 2 .

\section{References}

1 Brenner BM (ed). Pathogenesis of renal disease. In: Brenner and Rectors. The Kidney, Vol 1, 7th ed. WB. Saunders: London, 2004, pp 1322-1327.

2 Duvall-Young J, MacDonald MK, McKechnie N. Fundus changes in (type 2) glomerulonephritis simulating drusen: a histological report. Br J Ophthalmol 1989; 73: 297-302.

3 Duvall-Young J, Short CD, Raines MF, Gokal R, Lawler L. Fundus changes in mesangiocapillary glomerulonephritis type 2: clinical and fluorescein angiographic findings. $\mathrm{Br} \mathrm{J}$ Ophthalmol 1989; 73: 900-906.

4 Leys A, Proesmans W, Damme-Lombaerts RV, Van Damme B. Specific fundus lesions in type 2 membranoproliferative glomerulonephritis. Paediatr Nephrol 1991; 5(2): 189-192.
5 Kim DD, Mieler WF, Wolf MD. Posterior segment changes in membranoproliferative glomerulonephritis. Am J Ophthalmol 1992; 114(5): 593-599.

6 Michielsen B, Leys A, Van Damme B, Missotten L. Fundus changes in chronic membranoproliferative glomerulonephritis type 2. Doc Ophthalmol 1991; 76: 219229.

7 Gass JDM, Slamovits TL, Fuller DG, Gieser RG, Lean JS. Posterior chorioretinopathy and retinal detachment after organ transplantation. Arch Ophthalmol 1992; 110: 1717-1722.

8 Mullins RF, Russell SR, Anderson DH, Hagemann GS. Drusen associated with aging and age-related macular degeneration contain proteins common to extracellular deposits associated with atherosclerosis, elastosis, amyloidosis, and dense deposit disease. FASEB J 2000; 14(7): 835-846.

9 Leys A, Vanrenterghem Y, Van Damme B, Snyers B, Pirson L, Leys M. Fundus changes in membranoproliferative glomerulonephritis type 2. A fluorescein angiographic study of 23 patients. Graefe's Arch of Clin Exp Ophthalmol 1991; 229(5): 406-410.

10 Ulbig MRW, Riordan-Eva PR, Holz FG, Rees HC, Hamilton PAM. Membranoproliferative glomerulonephritis type 2 associated with central serous retinopathy. Am J Ophthalmol 1993; 116: 410-413.

11 Polk TD, Kimura AE, Park D, Gass JDM. Subretinal fluid associated with membranoproliferative glomerulonephritis type 2. Arch Ophthalmol 1997; 115: 927-928.

12 Leys A, Van Damme B, Verberckmoes R. Ocular complications of type 2 membranoproliferative glomerulonephritis. Nephrol Dial Transplant 1996; 11(1): 211-214.

13 Leys A, Michielsen B, Leys M, Vanrenterghem Y, Missotten L, Van Damme B. Subretinal neovascular membranes associated with chronic membranoproliferative glomerulonephritis type 2. Graefe's Arch Clin Exp Ophthalmol 1990; 228(6): 499-504. 